\title{
A Retrospective Analysis of Spinal Canal Endoscopy and Laminectomy Outcomes Data
}

\author{
Lloyd R. Saberski, MD
}

Disability from back pain has continued to increase at a steady pace since the 1950 's. While no study has associated chronic disability with physical factors, numerous studies have correlated chronic disability with psychological factors and surgical intervention.

Even though most common surgical interventions for spinal pain pertain to the herniated disc, numerous studies have indicated that only $1 \%$ of severe episodes of low back pain is attributable to herniated discs. With the advent of spinal canal endoscopy, an additional option is available for medical management of disc disease, which facilitates irrigation, dilution, and removal of inflammatory mediators, thus decreasing the chance of reactivity to chemical and biological mediators, and facilitating administration of corticosteroid medication with specificity to site of action.
This pilot study included two groups of patients, Group 1 with 22 patients treated via spinal endoscopy and Group 2 with 13 patients treated via laminectomy. After spinal canal endoscopy, only $31.8 \%$ of Group 1 patients were continued on opioid medication, whereas, $92.3 \%$ of Group 2 patients were continued on opioid medication after laminectomy. In addition, $72 \%$ from spinal canal endoscopy group and only $28 \%$ from laminectomy group returned to work.

In conclusion, this study suggested remarkable differences in outcomes when comparing patients who underwent spinal canal endoscopy to a similar population who underwent lumbar laminectomy.

Keywords: Spinal canal endoscopy, lumbar laminectomy, herniated disc, low back pain.
There has been no demonstrable increase in the prevalence or severity of low back pain in the general population for 40 years. Despite this plateau, several studies have shown that disability from back pain has increased at a steady pace since the 1950s $(1,2)$. Interestingly, no study has associated chronic disability with physical factors such as height, weight, mobility, strength, or severity of injury (3). In contrast, there are numerous studies correlating chronic disability with psychosocial factors such as anxiety, depression, drug and alcohol abuse, low job satisfaction, poor job performance, and altered family dynamics (4-8). Surprisingly, a factor strongly associated to chronic disability is surgical intervention $(2,9,10)$. A well-designed study from Oregon related rising costs in Workers' Compensation to high rates of surgical failure (11).

From Advanced Diagnostic Pain Treatment Centers, PC., Yale - New Haven Hospital, New Haven, Connecticut. Dr. Saberski is medical director of Advanced Diagnostic Pain Treatment Centers, PC. Address correspondence: Lloyd R. Saberski, MD, 60 Temple Street, Suite 4D, New Haven, CT 06510
The most common surgical interventions for spinal pain pertain to the herniated disc. However, numerous studies have indicated that only $1 \%$ of severe episodes of low back pain is attributable to herniated discs. The associated sciatica is usually self-limited and resolves with conservative care in $80 \%$ to $85 \%$ of the cases (12-16). Even in those patients with a herniated disc with neurologic deficit (numbness and weakness), there is equal resolution in conservatively treated patients compared with surgically managed ones $(13,14)$. A large-scale English study showed that $86 \%$ of patients with herniated disc with sciatica had good outcome with conservative, nonsurgical treatment (16). Another study demonstrated that $83 \%$ of patients advised to have urgent surgery could avoid surgical intervention and still achieve a good-to-excellent outcome (15). Even more fascinating was the fact that the discs most suitable to surgery showed the greatest tendency towards regression on follow-up magnetic resonance imaging (MRI) scan. In other developed countries, surgery is utilized less and only when there is evidence of a cauda equina compression or multiradicular symptoms. As a result, outcome is better. On the strength of clinical and epidemiological studies as outlined above, we must recognize that only between 15 to 50 of every 10,000 cases 
of acute low back pain should require surgery, while the remaining cases should be managed with conservative care. Thus, low back pain qualifies as a medical disease and not necessarily a surgical problem, even in cases of herniated disc.

To date, medical management for disc-related phenomena is the use of oral medications and exercise programs. With the advent of spinal canal endoscopy, additional options are now available for medical care of disc disease. Spinal canal endoscopy represents a platform that allows for the medical management of disc-related inflammation in the spinal canal. McCarron established that disc material, when placed into the spinal canal of dogs, causes an inflammatory response (18). This response is initiated by various inflammatory mediators including Phospholipase A. The patient's immune system will continue to respond in the presence of inflammatory mediators.

Spinal canal endoscopy, as currently practiced, can irrigate, dilute, and remove inflammatory mediators, decreasing the chance of reactivity to chemical and biologic mediators. In addition, spinal canal endoscopy can direct corticosteroid medication with specificity to site of action. Such directed injection serves to suppress components of the inflammatory response and becomes the first chemotherapy targeting disc-related inflammatory response.

\section{MATERIALS AND METHODS}

Data collection was performed within one geographical area of the country. The initial sample population consisted of 35 patients, ages 35 to 55 years, with radicular spinal pain determined through history, physical examination, and MRI. The patients were divided into two groups: Group $1(\mathrm{n}=22)$ was treated via spinal endoscopy and Group $2(\mathrm{n}=13)$ was treated via laminectomy. Group 1 patients were treated with 8 weeks of physical therapy and oral analgesic medication prior to enrollment for spinal canal endoscopy. Group 2 patients were treated with 8 weeks of physical therapy, oral analgesic medication, and lumbar and caudal epidural steroid injections before data collection. While there were additional facets to the treatment regimes, this report restricts itself to the statistical analysis of the results.

Basic descriptive statistics consisted of generating contingency tables. Tables are arranged for ready comparison of each group's response. Preprocedure is self-explanatory. Postprocedure is an 8-week follow-up. Demographic data were not complete from the presented data. Hence, no analysis or correlation was conducted insofar as demographic factors were concerned.

This report consists of the results of statistical analysis of procedural response only, even though data is presented without exclusion. Multiple variables including demographic factors have not been analyzed. Any conclusions drawn beyond the presented data is at the discretion of the reader.

\section{RESULTS}

Data analysis compared the two groups for the significance of their responses to opiate use and return-to-work rates following their respective procedures (Tables 1 and 2). Neuromedication use was not tested due to the $100 \%$ response rates following each group's respective procedure. Figure 1 illustrates postprocedure opiate usage. The chi-square analysis was performed using summarized data with two nominal variables (procedure at two levels and response at two levels for each procedure). Using a sig-

Table 1. Contingency Table for Opiate Usage Rate Comparison

\begin{tabular}{c|cc|cc|cc|cc}
\hline \multirow{2}{*}{ Contingency } & \multicolumn{3}{c|}{ Spinal Endoscopy } & \multicolumn{3}{c}{ Open Laminectomy } \\
\cline { 2 - 8 } & Preprocedure & Postprocedure & Preprocedure & Postprocedure \\
\cline { 2 - 8 } & Count & Rate & Count & Rate & Count & Rate & Count & Rate \\
\hline No & 8 & $36.4 \%$ & 15 & $68.2 \%$ & 0 & $0 \%$ & 1 & $7.7 \%$ \\
Yes & 14 & $63.6 \%$ & 7 & $31.8 \%$ & 13 & $100 \%$ & 12 & $92.3 \%$ \\
Totals & 22 & $100 \%$ & 22 & $100 \%$ & 13 & $100 \%$ & 13 & $100 \%$ \\
\hline
\end{tabular}


Table 2. Contingency Table for Return-to-Work Rates

\begin{tabular}{c|cc|cc}
\hline \multirow{2}{*}{ Contingency } & \multicolumn{2}{|c|}{ Spinal Endoscopy } & \multicolumn{2}{c}{ Open Laminectomy } \\
\cline { 2 - 5 } & Count & Rate & Count & Rate \\
\hline No & 6 & $27.3 \%$ & 9 & $69.2 \%$ \\
Yes & 16 & $72.7 \%$ & 4 & $30.8 \%$ \\
Totals & 22 & $100 \%$ & 13 & $100 \%$ \\
\hline
\end{tabular}

nificance level of 0.05 , the responses are significantly independent between treatment groups. A similar approach was used to compare the return-to-work rates (Fig. 2).

In Group 1, 14 of the 22 patients were receiving opioid medication pre-procedure. In Group 2, all 13 patients were receiving opioid medication preprocedure. After spinal canal endoscopy (Group 1) 7 patients (31.8\%) continued on opioid medication. After laminectomy (Group 2) 12 patients $(92.3 \%)$ continued on opioid medication.

Neuropathic medication was used in all 22 patients from Group 1 before and after spinal canal endoscopy. In Group 2 , three patients were on neuropathic medication preprocedure, and 13 were on neuropathic medication postprocedure.

Sixteen patients (73\%) from Group 1 returned to work and four patients (27\%) from Group 2 returned to work.

Fig. 1. Graph Showing Postprocedure Opiate

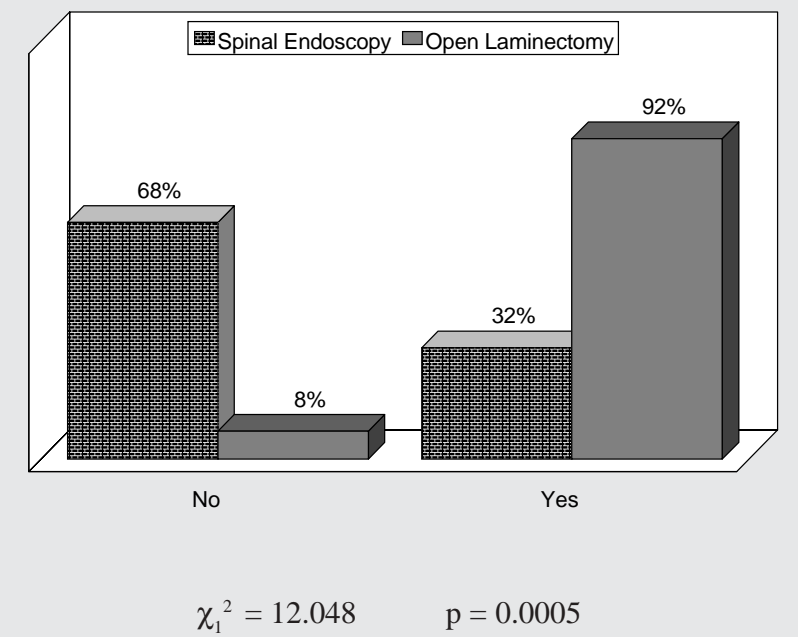

\section{DISCUSSION}

A relatively small number of postlaminectomy patients returned to work compared to the medically managed spinal canal endoscopy group. The reasons for this are multifaceted: (1) the nontraumatic nature of spinal canal endoscopy compared to laminectomy; (2) the short recovery times of spinal canal endoscopy compared to laminectomy; and (3) the medical management of patients (nonsurgical candidates) using several modalities including medication and spinal canal endoscopy. Although similar retrospective and preliminary data have been collected before and have indicated that spine disability is associated with laminectomy $(2,9,10)$, these data suggest that avoidance of laminectomy and adherence to a medical protocol featuring spinal canal endoscopy are associated with high return to work. This represents a substantial savings in health care and disability expenditure. The cost savings, when extrapolated to an entire population, suggest that substantial dollars can be saved by adherence to medical programs that emphasize medical care and spinal endoscopy. It is estimated that one year of disability costs the system $\$ 15,171$ (18). Disability savings alone (not even considering costs of surgery, hospitalization, and recovery) on 100 patients can exceed one million dollars. Add to this, the savings related to not performing surgery and the total savings are many millions of dollars.

\section{CONCLUSION}

This pilot study suggests a remarkable difference in outcomes when comparing medically managed patients with the benefit of spinal canal endoscopy to a similar popula-

Fig. 2. Graph Showing Postprocedure Return-to-Work Rates

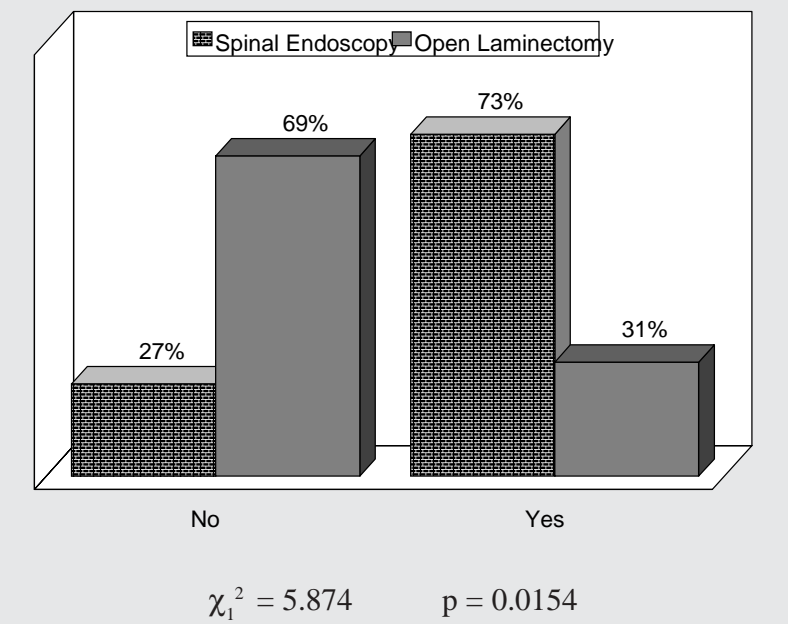


tion of postlaminectomy patients. A prospective study is now required to quantify the outcomes and cost savings of spinal canal endoscopy compared to laminectomy.

\section{REFERENCES}

1. Social Security Bulletin. Annual Statistical Supplement. Washington, D.C., US Government Printing Office, 1986.

2. Waddell G. A new model for the treatment of lowback pain. Volvo award in clinical sciences. Spine 1987; 12:632-644.

3. Rybock JD. Industrial low back pain. In Bleecker ML (ed). Occupational Neurology and Clinical Neurotoxicology. Baltimore, Williams and Wilkens, 1994; pp 335-343.

4. Bigos SJ, Battie MC, Sprengler NM et al. In Weinstein JN, Wiesel SW (eds). The Lumbar Spine. Philadelphia, WB Saunders, 1990; pp 846-859.

5. Helander E. Back pain and work disability. Social Medicine Times 1973; 50:398-404.

6. Lee PWJ, Chow SP, Lieh-Mak F et al. The psychosocial factors influencing outcomes in patients with low back pain. Spine 1989; 14: 838-846.

7. Magora A. Investigation of the relation between low back pain and occupation. 5 psychological aspects. Scand J Rehabil Med 1973; 5:186-190.

8. Waddell G, Mani CJ, Morris EW et al. Chronic low back pain, psychological distress and illness behavior. Spine 1984; 9: 209-213.

9. Frymoyer JW, Cats-Baril WL. An overview of the incidence and costs of low back pain. Orthop Clinics of North America 1991; 22: 263-271.

10. Frymoyer JW, Rosen JC, Clements J et al. Psychologic factors in low back pain disability. Clin Orthop 1985; 195: 178-184.

11. Norton WL. Chemonucleolysis versus surgical discectomy. Comparison of costs and results in workers' compensation claimants. Spine 1986; 11:440-443.

12. Hakelius A. Prognosis in sciatica. A clinical followup of surgical and non-surgical treatment. Acta Orthop Scand (suppl) 1970; 129:1-76.

13. Javid MJ, Nordby EJ, Ford LT. Safety and efficacy of chymopapain in herniated nucleus with sciatica. Results of a randomized double blind study. JAMA 1983; 249:2489-2494.

14. Weber H. Lumbar disc herniation. A controlled prospective study of 10 years of observation. Spine 1983; 8: 131-139.

15. Saal JA, Saal JS. Non-operative treatment of herniated intervertebral discs with radiculopathy. An outcome study. Spine 1989; 14: 431-437.

16. Bush K, Cowan N, Katz DE et al. The natural history of sciatica associated with disc pathology. Spine 1991; 17: 1205-1212.

17. McCarron RF. Epidural fibrosis. Experimental model and therapeutic alternatives. In Racz GB (ed). Techniques of Neurolysis. Boston, Kluwer Academic Press, 1989; pp. 87-94.

18. Turk Dc, Rudy TE, Stieg RL. The disability determination dilemma: towards a mutliaxial solution. Pain 1988; 34:217-29 